\author{
Johanna K Ecke², Luis Del Valle', \\ Amanda Parker Struckhoff ${ }^{1}$, Piotr \\ Waligorski ${ }^{1}$, Krzysztof Reiss ${ }^{1}$ and \\ Francesca Peruzzi ${ }^{1 *}$
}

${ }^{1}$ LSU Health Sciences Center School of Medicine and Stanley S Scott Cancer Center, New Orleans, USA

${ }^{2}$ Magazine Street Animal Clinic, New Orleans, USA

Dates: Received: 28 August, 2015; Accepted: 14 October, 2015; Published: 16 October, 2015

*Corresponding author: Francesca Peruzzi, LSUHSC School of Medicine and Stanley S Scott Cancer Center, 1700 Tulane Avenue, New Orleans, USA, Tel.: (504) 210-2978; Fax: (504) 210-2970; E-mail: fperuz@Isuhsc.edu

www.peertechz.com

Keywords: Dog; Fenofibrate; Hemangiosarcoma; Spleen

\author{
Case Report
}

\section{Increased Survival after Treatment of Canine Hemangiosarcoma with Fenofibrate}

\section{Abbreviations}

HSA: Hemangiosarcoma; PPAR- $\alpha$ : Peroxisome Proliferator Activated Receptor $\alpha$; MCV: Mean Corpuscular Volume; HPLC: HighPerformance Liquid Chromatography; ECG: Electrocardiogram; VPCs: Ventricular Premature Complexes;

\section{Introduction}

Hemangiosarcoma (HSA) is an incurable tumor of endothelial cell origin. The most common primary sites of this tumor are the spleen, the right atrium of the heart and the subcutis [1]. Visceral hemangiosarcomas, which often develop in the spleen or in the right heart base, account for $2 \%$ of all reported malignancies and up to $5 \%$ of all non-cutaneous tumors in dogs [2]. While occasionally dogs can survive longer than 12 months [3], the average survival time is 1-3 months after surgical removal of the tumor and 3-6 months with conventional chemotherapy after surgery [4].

The peroxisome proliferator activated receptor $\alpha(P P A R \alpha)$ agonist fenofibrate is a commonly used lipid-lowering human drug. The recommended daily dose to lower triglycerides and cholesterol in the blood in humans is $200 \mathrm{mg}$. In one study, fenofibrate treatment (10 $\mathrm{mg} / \mathrm{kg}$ daily for 2 weeks) efficiently lowered lipid parameters in obese dogs [5]. Several laboratories, including ours, have demonstrated the anti-cancer and anti-metastatic properties of fenofibrate in cultured cells as well as in small animal models [6-20]. Long-term treatment with fenofibrate at doses of 12 or 40 times the human dose induces liver carcinoma in rodents, but has not been shown to have an adverse effect in other species [21,22]. Increased creatininemia has been observed in human patients following long-term fenofibrate treatment [23-25]; however, a recent study demonstrated reversibility of renal dysfunction after 50 days off-drug [23-25]. As hemangiosarcoma is a very aggressive tumor with a high rate of metastasis and poor prognosis and fenofibrate is a well-tolerated drug, we elected to treat one case of canine HSA with fenofibrate.

\section{Case Presentation}

A 6.5-year-old spayed female white Boxer presented for evaluation at a veterinary clinic following two intermittent episodes of lethargic behavior and inappetence. A physical examination revealed a slightly tender cranial abdomen and possible cranial abdominal organomegaly; abdominal ultrasound discovered a hypoechoic splenic mass measuring 3-4 $\mathrm{cm}^{2}$. Complete blood count and biochemistry profile were within normal limits, except for a slightly elevated MCV (mean corpuscular volume). Splenectomy was performed the following day and histopathology of the tumor identified a low-grade hemangiosarcoma (mitotic index 4, Figure 1). At the time of surgery, a possible liver nodule was noted but not removed. Following diagnosis and splenectomy, the dog was administered oral fenofibrate at a dose of $400 \mathrm{mg} /$ day $(13 \mathrm{mg} / \mathrm{kg})$. Note that this dose was close to the dose of $10 \mathrm{mg} / \mathrm{kg}$ utilized in a previous study demonstrating that fenofibrate treatment lowers lipids in the blood of obese dogs [5]. During course of the fenofibrate treatment, the dog behaved normally, willingly participated in all daily routines, had a good appetite and did not show any sign of pain or discomfort. Routine physical examination performed eleven months after splenectomy revealed punctate, dermal masses at multiple locations on the patient's ventrum. A red dermal mass of about $2 \mathrm{~mm}$ in diameter was noted at the right axilla. The remainder of the physical exam was within normal limits and no evidence of metastatic disease was detected on thoracic radiographs and abdominal ultrasound. The patient did not show side effects related to fenofibrate treatment, except for increased appetite. Complete blood count and biochemistry profile remained within normal limits. At this time, high-performance liquid chromatography (HPLC) was performed on urine samples and the presence of high levels of the fenofibrate metabolite, fenofibric acid, was confirmed (Figure 2) and 


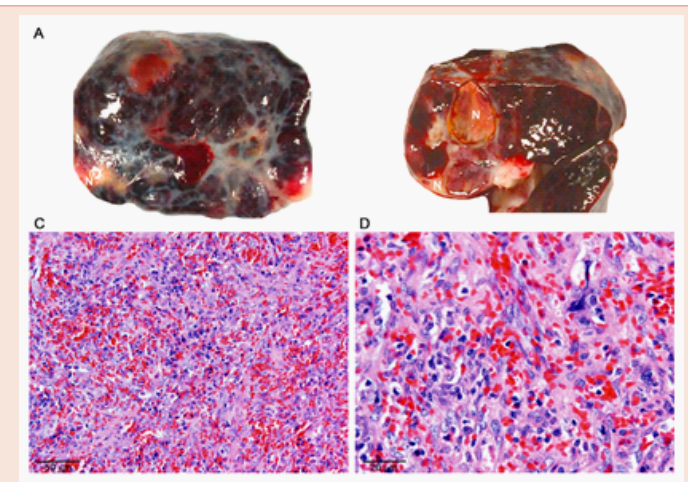

Figure 1: Histopathology of original tumor. Grossly the tumor was a welldefined, partially encapsulated, irregularly shaped mass with a nodular pattern (Panel A). Sections revealed a highly hemorrhagic tumor with areas of necrosis (Panel B). Histologically, the tumor is characterized by abundant pleomorphic irregularly shaped cells that form vascular channels filled with erythrocytes (Panel C, H\&E, and Original magnification $400 \mathrm{x}$ ). At higher magnification, neoplastic cells show pleomorphic nuclei with extended chromatin and some a prominent nucleoli and an abundant eosinophilic cytoplasm. Scarce mitotic figures were found (Panel D, H\&E, Original magnification 1000x). The letter $N$ in panel A stands for necrosis.

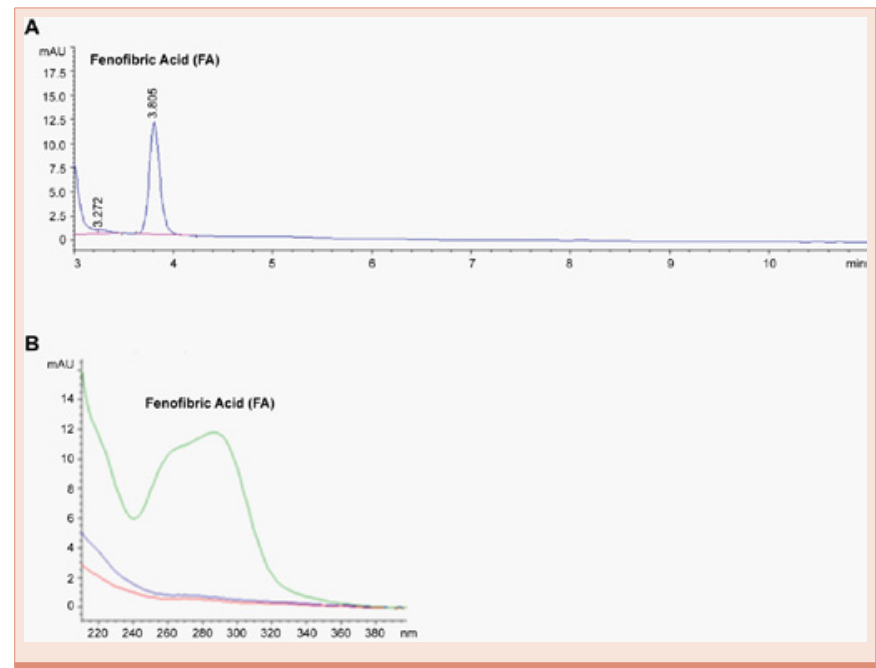

Figure 2: HPLC detection of fenofibric acid. A) Fenofibric acid (FA), the major metabolite of fenofibrate (FF), is present in the urine sample after 360 days of daily administration of FF. We used Agilent Technologies 1100 HPLC with on line degasser, binary pump, auto-sampler, YMC Base, $3 \mu \mathrm{m}$, $4.6 \times 150 \mathrm{~mm}$ column, thermostat, and diode array detector (DAD). The sample separation parameters were: solvent $A: 50 \mathrm{mM}$ acetic acid in water, solvent B: acetonitrile, isocratic $60 \%$; flow rate $1 \mathrm{ml} / \mathrm{min}$; temp $20^{\circ} \mathrm{C}$; injection fraction $5 \mu l$; detection: DAD at $285 \mathrm{~nm}$. Under the described conditions fenofibric acid was eluted at 3.8 minutes and fenofibrate at $10.2 \mathrm{~min}$. As expected, we did not detect FF in the urine sample. B) spectral analysis of the obtained peak corresponds to fenofibric acid (UV/Vis absorbance spectra max $=280 \mathrm{~nm}$ ), as we previously reported [17].

matched with the data obtained from blood and urine samples of human patients taking fenofibrate regularly [17].

After an additional eleven months (twenty-two months after initial diagnosis of splenic HSA), the dog presented with unusual behavior possibly associated with pain and distress, one episode of lethargy, vomiting, diarrhea and reduced appetite. On physical examination, the dog was bright, alert and responsive. Her mucous membranes were pink. No peripheral lymphadenopathy was noted, and thoracic auscultation was within normal limits. Abdominal palpation was non-painful, and revealed a possible soft tissue mass in the region of the right kidney. Several small, raised, red skin masses were noted. The largest skin mass $(1 \mathrm{~cm})$ was the one previously noted at the right lateral thorax just caudal to the elbow. Abdominal ultrasound confirmed an approximately $4 \times 7 \mathrm{~cm}$ cavitary mass of mixed echogenicity just caudal to the right kidney. There were no additional masses identified in the abdomen. The liver was difficult to evaluate due to her deep chest, but no obvious masses were seen. There was no evidence of abdominal effusion. Thoracic and abdominal radiographs were performed and no evidence of metastatic disease was found. Complete blood count, biochemistry profile and urinalysis were within normal limits. An ECG showed a respiratory sinus arhythmia (no VPCs). An echocardiogram showed no significant abnormalities and no evidence of a heart-based tumor. After surgical removal of the perirenal tumor, histopathology results identified the mass as an intermediate-grade hemangiosarcoma (mitotic index 6, Figure 3). Adjacent tissue, including a portion of the kidney capsule, was negative for neoplastic cells. The dog recovered well from the surgery and the daily dose of fenofibrate was increased by $1 / 3$ to achieve a total dose of $600 \mathrm{mg} /$ day $(19 \mathrm{mg} / \mathrm{kg}$ ). Four months later (twenty-six months after initial diagnosis), the dog presented with lethargy and pain; 12 hours later she developed tetraparesis with upper motor neuron signs in all four limbs. On physical exam, the patient exhibited moderate to severe pain over the thoracolumbar spinal region and moderate abdominal pain. She also exhibited pain on palpation of the cervical spine and decreased cervical range of motion when turning her head to the left. The mass on the right axilla was bleeding intermittently. Complete blood count showed a slightly low packed cell volume; biochemistry profile was normal except for a mild hypernatremia and hyperchloremia, and a slightly elevated total protein. Abdominal radiographs were normal. Spinal radiographs revealed moderate spondylosis at L2-4, and mild spondylosis at T10-L1. Based on the patient's clinical signs, a cervical central nervous system lesion was suspected. Top differentials included primary or metastatic neoplasia, intervertebral disc disease, infection (meningitis), and inflammation.

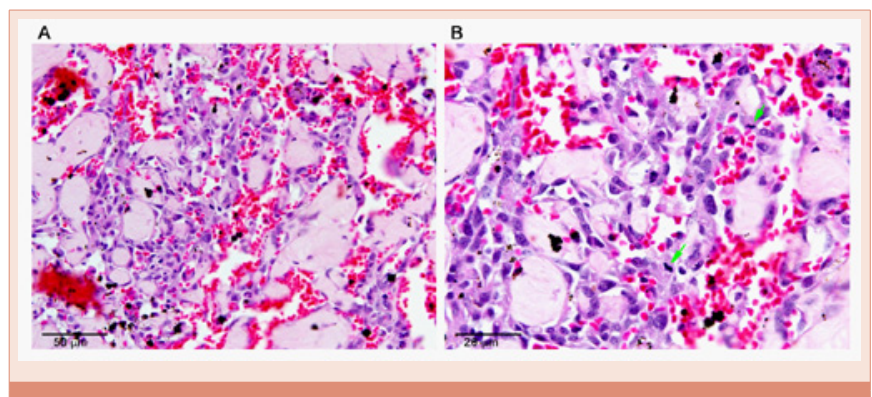

Figure 3: Histopathological features of recurrence. As the original tumor, the recurrence is characterized by numerous irregular neoplastic cells that form vascular channels. However, these tumor cells now sit on a highly fibrous core (Panel A, H\&E, Original magnification 400x). At higher power, neoplastic cells are smaller than the original tumor and have darker, hyper chromatic nuclei. Numerous mitotic figures are present (green arrows). The highly fibrous stroma is evident (Panel B, H\&E, Original magnification 1000x). 
Due to the patient's severe clinical signs, rapid decline, and history of hemangiosarcoma, no additional diagnostics (advanced imaging, cerebrospinal fluid analysis) were performed. After a short period of conservative management with continued decline, the patient was humanely euthanized.

\section{Conclusion}

Considering the aggressiveness of canine hemangiosarcoma and the reported anti-cancer activities of fenofibrate [9,11-15,17,20], we believe that fenofibrate treatment, likely together with the early detection of the tumor, delayed recurrence of the tumor as well as formation of metastases. Since fenofibrate cannot cross the blood-brain barrier and reach the central nervous system [17], it is possible that metastatic disease occurred in this organ contributing to the end-stage paralysis and death, although this assumption was never confirmed. While additional studies are required to confirm the effectiveness of fenofibrate in the treatment of canine hemangiosarcoma, and perhaps other aggressive tumors, there are a few important considerations worth mentioning: 1) fenofibrate is a common drug already approved for hyperlipidemia, therefore it is highly accessible; 2) the dosage utilized for this case of HSA and for reducing lipid parameters in obese dogs was similar (13 versus $10 \mathrm{mg} / \mathrm{kg}$ ); 3) fenofibrate is easy to administer orally by dog owners and does not require visits to the veterinary clinic; 4) it is relatively inexpensive; and 5) it does not appear to cause discomfort or evident adverse effects.

\section{Acknowledgements}

We thank Dr. Lea C Disston for early diagnosis of the tumor and splenectomy, and Drs. Elizabeth and David Kergosien for the surgery on the recurrent tumor. We also thank the entire staff at the Magazine Street Animal Clinic and at the Louisiana Veterinary Referral Center for their professionalism and remarkable support. A special thank you to Margo for being part of our lives (FP, KR).

\section{References}

1. Locke JE and Barber LG (2006) Comparative aspects and clinical outcomes of canine renal hemangiosarcoma. Journal of veterinary internal medicine / American College of Veterinary Internal Medicine 20: 962-967.

2. Smith AN (2003) Hemangiosarcoma in dogs and cats. Vet Clin North Am Small Anim Pract 33: 533-552.

3. Spangler WL, Kass PH (1997) Pathologic factors affecting postsplenectomy survival in dogs. Journal of veterinary internal medicine / American College of Veterinary Internal Medicine 11: 166-171.

4. Wood CA, Moore AS, Gliatto JM, Ablin LA, Berg RJ, et al. (1998) Prognosis for dogs with stage I or II splenic hemangiosarcoma treated by splenectomy alone: 32 cases (1991-1993). J Am Anim Hosp Assoc 34: 417-421.

5. Serisier S, Briand F, Ouguerram K, Siliart B, Magot T, et al. (2006) Fenofibrate lowers lipid parameters in obese dogs. J Nutr 136: 2037S-2040S.

6. Chang NW, Tsai MH, Lin C, Hsu HT, Chu PY, et al. (2010) Fenofibrate exhibits a high potential to suppress the formation of squamous cell carcinoma in an oral-specific 4-nitroquinoline 1-oxide/arecoline mouse model. Biochimica et biophysica acta 1812: 558-564.
7. Jiao HL and Zhao BL (2002) Cytotoxic effect of peroxisome proliferator fenofibrate on human HepG2 hepatoma cell line and relevant mechanisms. Toxicol Appl Pharmacol 185: 172-179.

8. Maiguma T, Fujisaki K, Itoh Y, Makino K, Teshima D, et al. (2003) Cellspecific toxicity of fibrates in human embryonal rhabdomyosarcoma cells. Naunyn Schmiedebergs Arch Pharmacol 367: 289-296.

9. Panigrahy D, Kaipainen A, Huang S, Butterfield CE, Barnes CM, et al. (2008) PPARalpha agonist fenofibrate suppresses tumor growth through direct and indirect angiogenesis inhibition. Proc Natl Acad Sci U S A 105: 985-990.

10. Urbanska K, Pannizzo P, Grabacka M, Croul S, Del Valle L, et al. (2008) Activation of PPARalpha inhibits IGF-I-mediated growth and survival responses in medulloblastoma cell lines. Int J Cancer 123: 1015-1024.

11. Wilk A, Urbanska K, Grabacka M, Mullinax J, Marcinkiewicz C, et al. (2012) Fenofibrate-induced nuclear translocation of FoxO3A triggers Bim-mediated apoptosis in glioblastoma cells in vitro. Cell cycle 11: 2660-2671.

12. Yamasaki D, Kawabe N, Nakamura H, Tachibana K, Ishimoto K, et al. (2011) Fenofibrate suppresses growth of the human hepatocellular carcinoma cell via PPARalpha-independent mechanisms. Eur J Cell Biol 90: 657-664.

13. Zak Z, Gelebart P and Lai R (2010) Fenofibrate induces effective apoptosis in mantle cell lymphoma by inhibiting the TNFalpha/NF-kappaB signaling axis. Leukemia 24:1476-1486.

14. Grabacka M, Placha W, Plonka PM, Pajak S, Urbanska K, et al. (2004) Inhibition of melanoma metastases by fenofibrate. Arch Dermatol Res 296: 54-58.

15. Grabacka M, Plonka PM, Urbanska K, Reiss K (2006) Peroxisome proliferator-activated receptor alpha activation decreases metastatic potential of melanoma cells in vitro via down-regulation of Akt. Clin Cancer Res 12: 3028-3036.

16. Jeansonne D, Pacifici M, Lassak A, Reiss K, Russo G, et al. (2013) Differential Effects of MicroRNAs on Glioblastoma Growth and Migration. Genes 4: 4664.

17. Grabacka M, Waligorski P, Zapata A, Blake DA, Wyczechowska D, et al. (2015) Fenofibrate subcellular distribution as a rationale for the intracranial delivery through biodegradable carrier. J Physiol Pharmacol 66: 233-247.

18. Jeansonne D, DeLuca M, Marrero L, Lassak A, Pacifici M, et al. (2015) Antitumoral Effects of miR-3189-3p in Glioblastoma. J Biol Chem 290: 80678080 .

19. Piwowarczyk K, Wybieralska E, Baran J, Borowczyk J, Rybak P, et al. (2015) Fenofibrate enhances barrier function of endothelial continuum within the metastatic niche of prostate cancer cells. Expert Opin Ther Targets 19: 163176.

20. Wilk A, Wyczechowska D, Zapata A, Dean M, Mullinax J, et al. (2015) Molecular mechanisms of fenofibrate-induced metabolic catastrophe and glioblastoma cell death. Mol Cell Biol 35: 182-198.

21. Roberts WC (1989) Safety of fenofibrate--US and worldwide experience. Cardiology 76: 169-179.

22. Fidaleo M (2009) Human health risk assessment for peroxisome proliferators: more than 30 years of research. Exp Toxicol Pathol 61: 215-221.

23. Hottelart C, El Esper N, Rose F, Achard JM, Fournier A (2002) Fenofibrate increases creatininemia by increasing metabolic production of creatinine. Nephron 92: 536-541.

24. Lipscombe J, Lewis GF, Cattran D, Bargman JM (2001) Deterioration in renal function associated with fibrate therapy. Clin Nephrol 55: 39-44.

25. Mychaleckyj JC, Craven T, Nayak U, Buse J, Crouse JR, et al. (2012) Reversibility of fenofibrate therapy-induced renal function impairment in ACCORD type 2 diabetic participants. Diabetes Care 35: 1008-1014.

Copyright: @ 2015 Peruzzi F, et al. This is an open-access article distributed under the terms of the Creative Commons Attribution License, which permits unrestricted use, distribution, and reproduction in any medium, provided the original author and source are credited. 